\title{
TWIN PARADOX FOR A REALISTICALLY ACCELERATING SPACE TRAVEL
}

\author{
Gábor Pszota \\ associate professor, Institute of Physics and Electrical Engineering \\ 3515 Miskolc, Miskolc-Egyetemváros, e-mail:fizpszo@uni-miskolc.hu \\ János Majár \\ associate professor, Institute of Physics and Electrical Engineering \\ 3515 Miskolc, Miskolc-Egyetemváros, e-mail:fizmajar@uni-miskolc.hu
}

\begin{abstract}
A common example for Einstein's special relativity [1] is the different aging of the twins, one of whom embarks on an interstellar journey. This problem was originally discussed with a spaceship moving at a constant speed there and back, and there was no mention of the accelerating interval. Without that, this situation appears to be paradoxical, since one can argue that the traveling twin also sees the other one moving, therefore the situation is symmetric. No such symmetry is apparent, however, if the interval of acceleration is considered, no matter how short that is [2,3]. We wanted to analyse this situation for a realistic scenario, where the spaceship would have a continuous acceleration and deceleration of $1 \mathrm{~g}$ during the entire round trip. We present detailed calculations and an analysis of the aging difference for journeys to various distances and for various times.
\end{abstract}

Keywords: special relativity, twin paradox, time dilation, interstellar travel

\section{Introduction}

In the nineteenth century the question arose whether there is a hypothetical medium called aether, relative to which the speed of light in vacuum should be measured. It is easy to imagine that if such a medium existed, it could be taken as an absolute stationary system. In any other system that moves compared to this system, the speed of light would appear different in the different directions, according to the Galilean transformation formulas. These formulas are easy to write up if we consider the stationary $S$ system and an $S$ ' system that moves along the positive $x$ axis of the $S$ system at some $v_{0}$ velocity. An object moves along a straight path at a constant speed, as it can be seen in Figure 1.

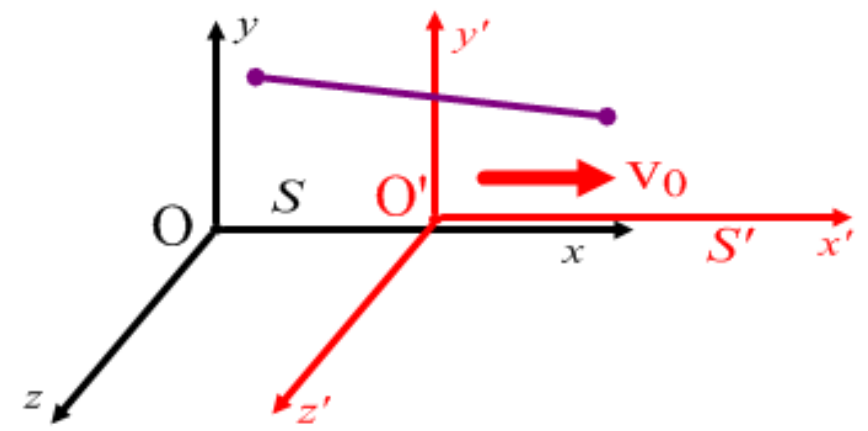

Figure 1. The stationary $S$ and the moving $S$ ' systems 
During some $\Delta t$ time measured in $S$ or $\Delta t^{\prime}$ time measured in $S^{\prime}\left(\Delta t=\Delta t^{\prime}\right)$, the coordinate differences in each system will be the following:

$$
\Delta x^{\prime}=\Delta x-v_{0} \Delta t \quad \Delta y^{\prime}=\Delta y \quad \Delta z^{\prime}=\Delta z
$$

Now if we divide by $\Delta t$ or $\Delta t$ ', we get the transformation formulas for the velocity components:

$$
v_{x}{ }^{\prime}=v_{x}-v_{0} \quad v_{y}{ }^{\prime}=v_{y} \quad v_{z}{ }^{\prime}=v_{z}
$$

Several experiments, however, demonstrated that the speed of light is the same in any direction and in any moving inertial reference system. One such experiment was Michelson's interferometric experiment, which would have been able to show a few $\mathrm{m} / \mathrm{s}$ difference in the speed of light measured in different directions. These findings disproved the existence of the aether, and lead to the formulation of special relativity by Einstein [1].

\subsection{The Lorentz transformation formulas}

Einstein concluded that the speed of light is the same in any inertial reference frame, and in any direction. No inertial system can be chosen as an absolute stationary system, the laws of physics can be written with similar formulas in any inertial system. This means that the formulas of the Galilean transformation cannot be correct for high velocities, and they are just approximations for velocities much smaller than the speed of light. The correct transformation formulas were given by Einstein in his 1905 paper [1]. The flowing requirements had to be satisfied by the mathematical relations between $\Delta t, \Delta x, \Delta y, \Delta z$ of $S$ and $\Delta t^{\prime}, \Delta x^{\prime}, \Delta y^{\prime}, \Delta z^{\prime}$, of $S^{\prime}$ :

1. Two events that happen simultaneously at the same location in one inertial system should happen simultaneously and at the same location in the other system too. This means that if $\Delta t=\Delta x$ $=\Delta y=\Delta z=0$ then $\Delta t^{\prime}=\Delta x^{\prime}=\Delta y^{\prime}=\Delta z^{\prime}=0$ as well.

2. The formulas transforming from $S$ to $S^{\prime}$ 'should be similar to the formulas transforming from $S^{\prime}$ to $S$. Because of this, only linear transformation formulas are possible.

3. For small velocities the formulas should give back the Galilean transformation formulas.

4. The speed of light should be the same in every inertial reference frame.

In order to satisfy these conditions, Einstein had to give up the notion that clocks in the moving reference frames are synchronized to each other $\left(\Delta t=\Delta t^{\prime}\right)$, meaning time is just as relative as the coordinate differences. Experimental proof to this fact has been shown in several experiments that involved atomic clocks flown on airplanes [4], measuring the difference in the half-life of relativistic particles [5, 6], or moving optical clocks at a few percent of the speed of light [7]. The relativistic formulas connecting the $S$ frame to the $S^{\prime}$ moving relative to it at a speed of $v$ along the positive $x$ axis:

$$
\Delta t^{\prime}=\frac{\Delta t-\frac{v}{c^{2}} \Delta x}{\sqrt{1-\frac{v^{2}}{c^{2}}}} \quad \Delta x^{\prime}=\frac{\Delta x-v \Delta t}{\sqrt{1-\frac{v^{2}}{c^{2}}}} \quad \Delta y^{\prime}=\Delta y \quad \Delta z^{\prime}=\Delta z
$$

If we want to transform the coordinate and time differences from the $S$ ' system to $S$, we have to write $-v$ instead of $v$ : 


$$
\Delta t=\frac{\Delta t^{\prime}+\frac{v}{c^{2}} \Delta x^{\prime}}{\sqrt{1-\frac{v^{2}}{c^{2}}}} \quad \Delta x=\frac{\Delta x^{\prime}+v \Delta t^{\prime}}{\sqrt{1-\frac{v^{2}}{c^{2}}}} \quad \Delta y=\Delta y^{\prime} \quad \Delta z=\Delta z^{\prime}
$$

\subsection{Transforming the velocity}

Since the letter $v$ is taken to denote the speed of the $S^{\prime}$ system relative to $S$, it is customary to use $\vec{u}$ and $\vec{u}^{\prime}$ to denote the velocity of objects in the corresponding systems. The components of this velocity can be written in the following way:

$$
\begin{array}{lll}
u_{x}{ }^{\prime}=\frac{\Delta x^{\prime}}{\Delta t^{\prime}}=\frac{\Delta x-v \Delta t}{\Delta t-\frac{v}{c^{2}} \Delta x}=\frac{\frac{\Delta x}{\Delta t}-v}{1-\frac{v \Delta x}{c^{2} \Delta t}}=\frac{u_{x}-v}{1-\frac{v u_{x}}{c^{2}}} & \text { or } & u_{x}=\frac{\Delta x}{\Delta t}=\frac{u_{x}{ }^{\prime}+v}{1+\frac{v u_{x}{ }^{\prime}}{c^{2}}} \\
u_{y}{ }^{\prime}=\frac{\Delta y^{\prime}}{\Delta t^{\prime}}=\frac{\Delta y}{\frac{\Delta t-\frac{v}{c^{2}} \Delta x}{\sqrt{1-\frac{v^{2}}{c^{2}}}}}=\frac{u_{y} \sqrt{1-\frac{v^{2}}{c^{2}}}}{1-\frac{v u_{x}}{c^{2}}} & \text { or } & u_{y}=\frac{\Delta y}{\Delta t}=\frac{u_{y}{ }^{\prime} \sqrt{1-\frac{v^{2}}{c^{2}}}}{1+\frac{v u_{x}{ }^{\prime}}{c^{2}}} \\
u_{z}{ }^{\prime}=\frac{\Delta z^{\prime}}{\Delta t^{\prime}}=\frac{\Delta z}{\frac{\Delta t-\frac{v}{c^{2}} \Delta x}{\sqrt{1-v^{2}}}}=\frac{u_{z} \sqrt{1-\frac{v^{2}}{c^{2}}}}{1-\frac{v u_{x}}{c^{2}}} & \text { or } & u_{z}=\frac{\Delta z}{\Delta t}=\frac{u_{z}{ }^{\prime} \sqrt{1-\frac{v^{2}}{c^{2}}}}{1+\frac{v u_{x}{ }^{\prime}}{c^{2}}}
\end{array}
$$

\subsection{Transforming the acceleration}

In order to transform the $x$ component of the acceleration, we have to use the differential form of the transformation formulas (e.g. $\Delta t \rightarrow d t$ ):

$$
\begin{gathered}
a_{x}{ }^{\prime}=\frac{d u_{x}{ }^{\prime}}{d t^{\prime}}=\frac{\frac{d u_{x}{ }^{\prime}}{d u_{x}} d u_{x}}{\frac{d t^{\prime}}{d t} d t}=\frac{\frac{1-\frac{v u_{x}}{c^{2}}+\left(u_{x}-v\right) \frac{v}{c^{2}}}{\left(1-\frac{v u_{x}}{c^{2}}\right)^{2}}}{\frac{1-\frac{v u_{x}}{c^{2}}}{\sqrt{1-\frac{v^{2}}{c^{2}}}}} a_{x}=\frac{a_{x}\left(1-\frac{v^{2}}{c^{2}}\right)^{\frac{3}{2}}}{\left(1-\frac{v u_{x}}{c^{2}}\right)^{3}} \\
a_{x}=\frac{d u_{x}}{d t}=\frac{\frac{d u_{x}}{d u_{x}{ }^{\prime}} d u_{x}{ }^{\prime}}{\frac{d t}{d t^{\prime}} d t^{\prime}}=\frac{a_{x}{ }^{\prime}\left(1-\frac{v^{2}}{c^{2}}\right)^{\frac{3}{2}}}{\left(1+\frac{v u_{x}{ }^{\prime}}{c^{2}}\right)^{3}}
\end{gathered}
$$

We are not going to write up the $y$ and $z$ components of the acceleration, since our spaceship will be traveling along the $x$ axis, and its acceleration will also be directed along that axis. Therefore this is the only component that we will need. 


\section{Discussion}

The spaceship starts from Earth and travels with a constant acceleration of $g$, measured in the frame of the ship. This is called the proper acceleration. Since the velocity of the ship is continuously changing, if we want to fix an inertial reference frame to the ship such that $u_{x}{ }^{\prime}=0$, this will have to be a different $S$ ' system in every moment: $v=u_{x}$, where $u_{x}$ is the velocity of the ship in $S$, i.e. relative to Earth. In all of these different $S^{\prime}$ systems it will be true that $a_{x}{ }^{\prime}=g$. The transformation formula in (6) will be simplified due to the fact that $u_{x}{ }^{\prime}=0$ in every moment:

$$
a_{x}(v)=a_{x}{ }^{\prime}\left(1-\frac{v^{2}}{c^{2}}\right)^{\frac{3}{2}}=g\left(1-\frac{v^{2}}{c^{2}}\right)^{\frac{3}{2}}
$$

As we can see (Figure 2), the acceleration of the ship in $S$ goes to zero as it approaches the speed of light. Here we use $\beta=\frac{v}{c}$ to give the velocity in speed of light units.

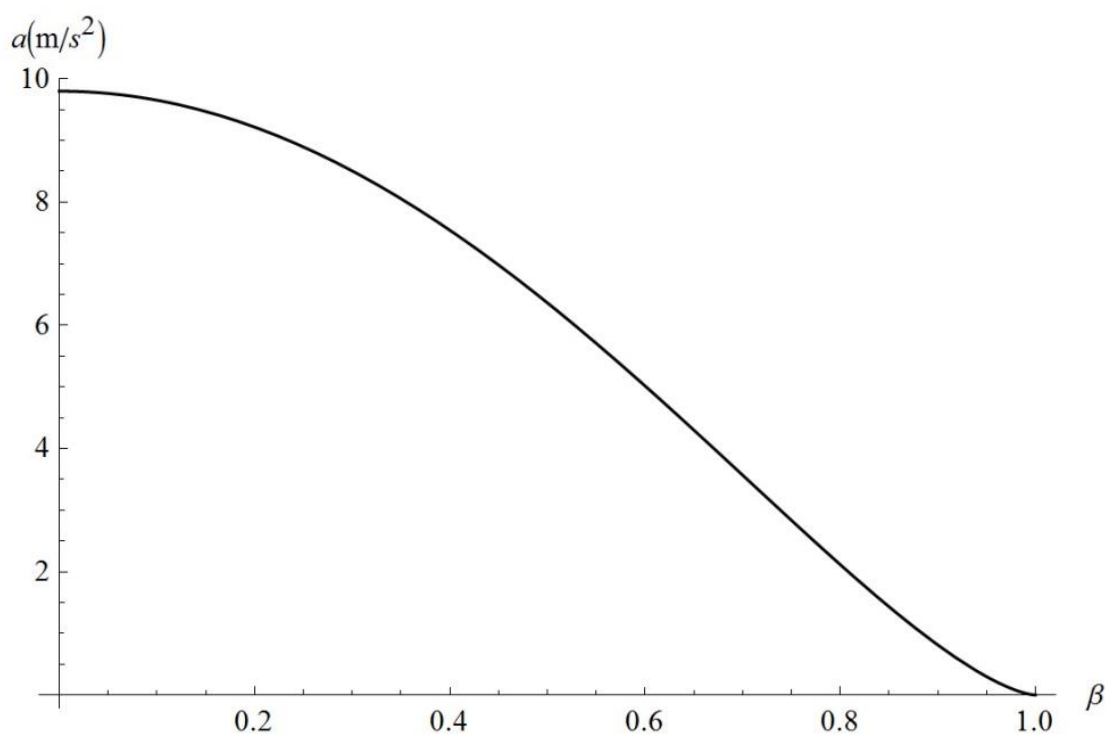

Figure 2. The acceleration of the ship as measured from Earth, as a function of its velocity

If we now substitute the time derivative of $u_{x}$ for $a_{x}$ in (7), and consider that due to the proper choosing of reference frames $u_{x}=v$ in every moment, we have a differential equation for $u_{x}$, which we can solve by separating the variables. We will use $\beta=v / c$ again:

$$
\begin{aligned}
& \frac{d v}{d t}=g\left(1-\frac{v^{2}}{c^{2}}\right)^{\frac{3}{2}} \\
& \frac{d(\beta c)}{d t}=g\left(1-\beta^{2}\right)^{\frac{3}{2}}
\end{aligned}
$$




$$
\begin{gathered}
\frac{d \beta}{d t}=\frac{g}{c}\left(1-\beta^{2}\right)^{\frac{3}{2}} \\
\frac{d \beta}{\left(1-\beta^{2}\right)^{\frac{3}{2}}}=\frac{g}{c} d t \\
\int_{0}^{\beta} \frac{d \beta^{\prime}}{\left(1-\beta^{\prime 2}\right)^{\frac{3}{2}}}=\int_{0}^{t} \frac{g}{c} d t^{\prime} \\
\frac{\beta}{\sqrt{1-\beta^{2}}}=\frac{g}{c} t
\end{gathered}
$$

We can solve this for $\beta$ and obtain the velocity in speed of light units as a function of time measured on Earth (see Figure 3 as well):

$$
\beta(t)=\frac{g t}{\sqrt{c^{2}+g^{2} t^{2}}}
$$

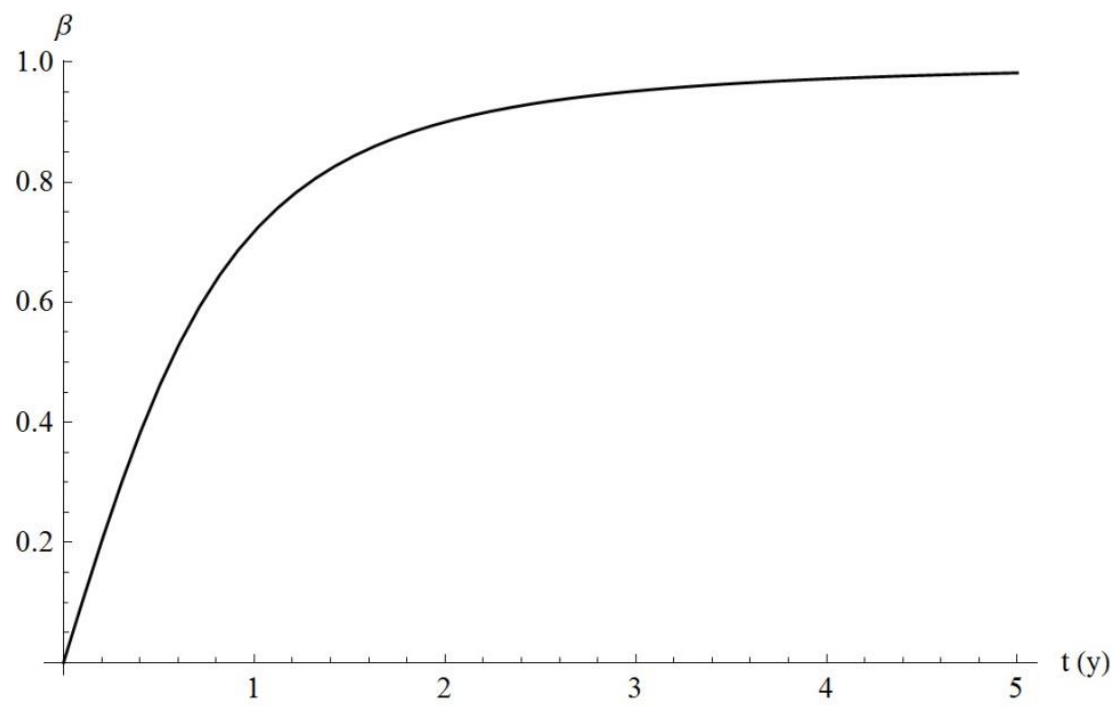

Figure 3. The speed of the ship as a function of time in years measured on Earth

We can see that the speed initially increases linearly according to Newtonian mechanics, but as we get close to the speed of light after spending almost a year accelerating at $g$ in the ship's reference frame, the acceleration as seen from Earth diminishes, and the ship can never reach the speed of light. If we plug (9) into (7), we get how this acceleration changes according to an observer on Earth (see Figure 4 as well): 


$$
a_{x}(t)=g\left(1-\frac{v^{2}}{c^{2}}\right)^{\frac{3}{2}}=g\left(1-\beta^{2}\right)^{\frac{3}{2}}=g\left(1-\frac{g^{2} t^{2}}{c^{2}+g^{2} t^{2}}\right)^{\frac{3}{2}}=g\left(\frac{c^{2}}{c^{2}+g^{2} t^{2}}\right)^{\frac{3}{2}}
$$

As we can see in Figure 4, the initial $1 g$ acceleration noticeably changes after a few months, and in a few years it drops to almost zero. This is consistent with the idea that the ship can approach the speed of light arbitrarily close, but it can never actually reach that value.

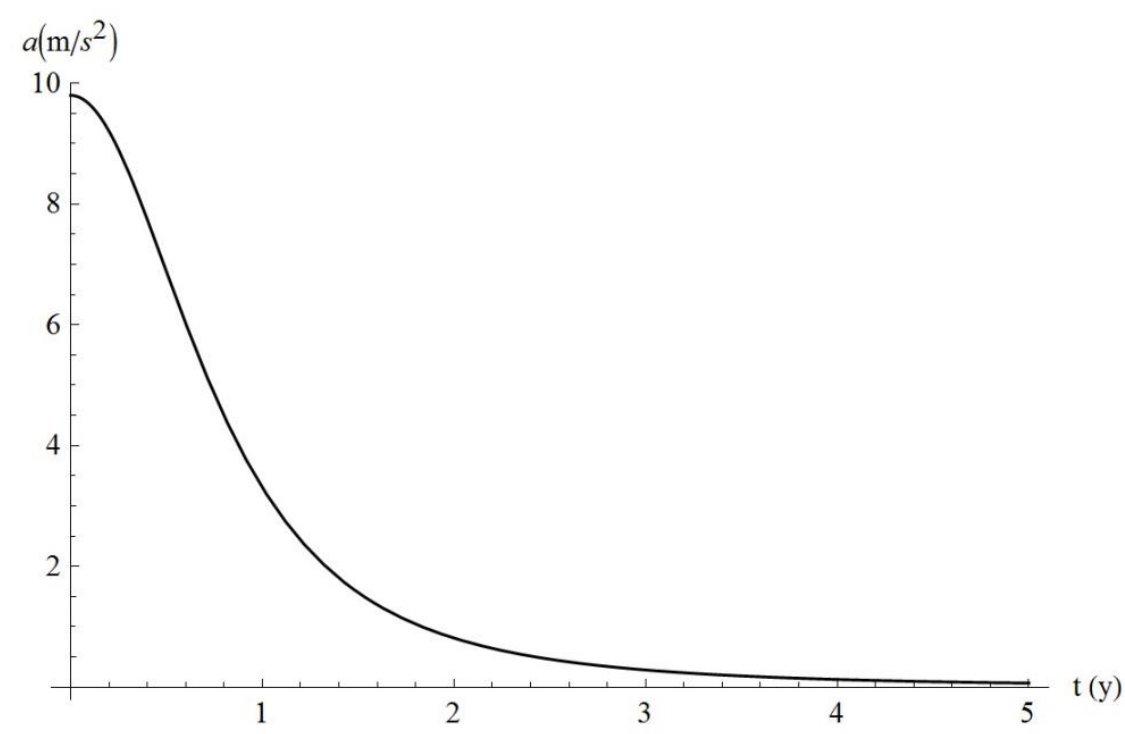

Figure 4. The acceleration of the ship as measured from Earth, as a function of time

If we now want to calculate how far the ship gets as a function of time, in (9) we can write $v$ as the time derivative of $x$, and calculate the integral:

$$
\begin{gathered}
v(t)=\frac{d x}{d t}=\beta c=\frac{g c t}{\sqrt{c^{2}+g^{2} t^{2}}}=\frac{g t}{\sqrt{1+\frac{g^{2}}{c^{2}} t^{2}}} \\
x(t)=\int \frac{g t}{\sqrt{1+\frac{g^{2}}{c^{2}} t^{2}}} d t+C_{1}=\frac{c^{2}}{g} \sqrt{1+\frac{g^{2}}{c^{2}} t^{2}}+C_{1}
\end{gathered}
$$

When $t=0, x=0$ :

$$
0=\frac{c^{2}}{g}+C_{1} \quad \rightarrow \quad C_{1}=-\frac{c^{2}}{g}
$$




$$
x(t)=\frac{c^{2}}{g} \sqrt{1+\frac{g^{2}}{c^{2}} t^{2}}-\frac{c^{2}}{g}=\frac{c^{2}}{g}\left(\sqrt{1+\frac{g^{2}}{c^{2}} t^{2}}-1\right)
$$

Looking at Figure 5, we can see that after the initial acceleration of a few months, the ship practically travels at the speed of light, and the distance just increases according to that. This way the closest star system can be reached in about 5 years if we do not plan on slowing down and stopping there.

Now let's see how the time measured on the ship compares to the time that they measure on Earth. We already have $t$ that we used to denote the time in $S$, and the proper time of the ship will have to be integrated over the infinite number of moving $S$ ' reference frames. Let's denote this time with the letter $\tau$.

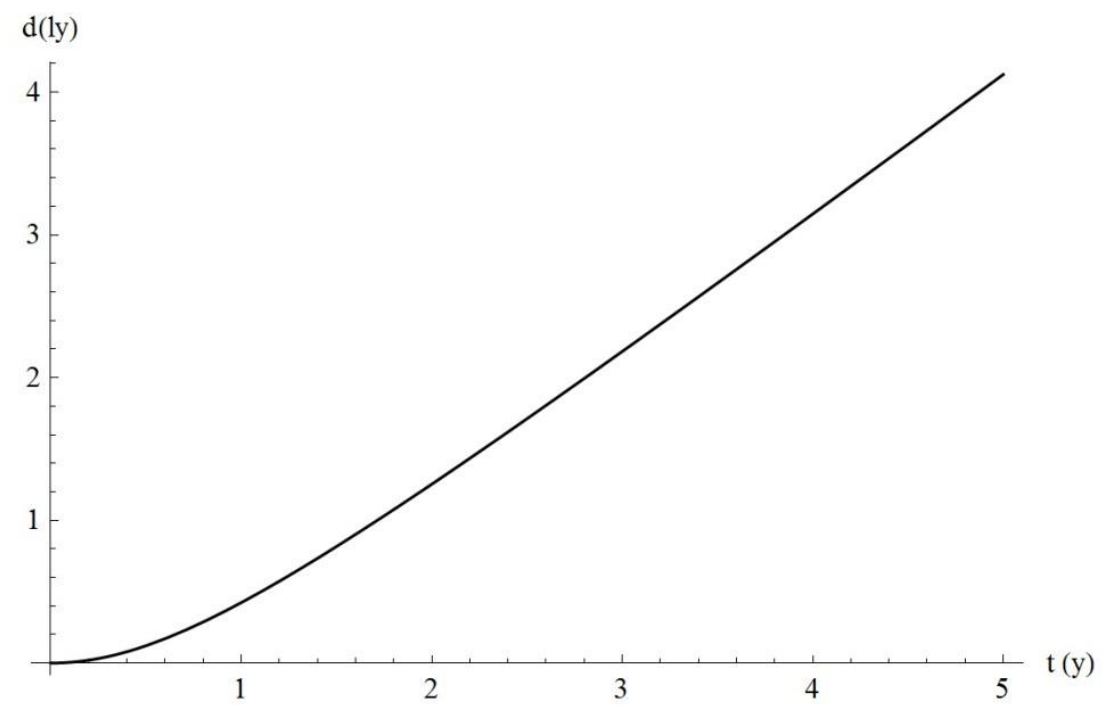

Figure 5. The distance from Earth in lightyears as a function of time

In the momentary $S$ ' system, which is moving at velocity $v=u_{x}$ with the ship, $d x^{\prime}$ is zero, therefore we can write the connection between $d t$ and $d t^{\prime}=d \tau$ using the Lorentz transformation formula for time from (4):

$$
d t=\frac{d \tau}{\sqrt{1-\frac{v^{2}}{c^{2}}}}
$$

After rearranging and plugging in what we got for $\beta$, we get a differential equation that we can solve for the proper time of the ship:

$$
\frac{d \tau}{d t}=\sqrt{1-\frac{v^{2}}{c^{2}}}=\sqrt{1-\beta^{2}}=\sqrt{\frac{c^{2}}{c^{2}+g^{2} t^{2}}}=\frac{1}{\sqrt{1+\frac{g^{2}}{c^{2}} t^{2}}}
$$




$$
\tau(t)=\int \frac{d t}{\sqrt{1+\frac{g^{2}}{c^{2}} t^{2}}}+C_{2}=\frac{c}{g} \sinh ^{-1}\left(\frac{g t}{c}\right)+C_{2}
$$

When $t=0$ then $\tau=0$ as well, therefore: $C_{2}=0$

$$
\tau(t)=\frac{c}{g} \sinh ^{-1}\left(\frac{g t}{c}\right)
$$

In Figure 6 we can see how the proper time of the ship depends on the time measured on Earth as the velocity increases and approaches the speed of light. For a few months there is not much difference, but after several years of acceleration the clock of the traveling twin slows down dramatically compared to the clock left with his brother.

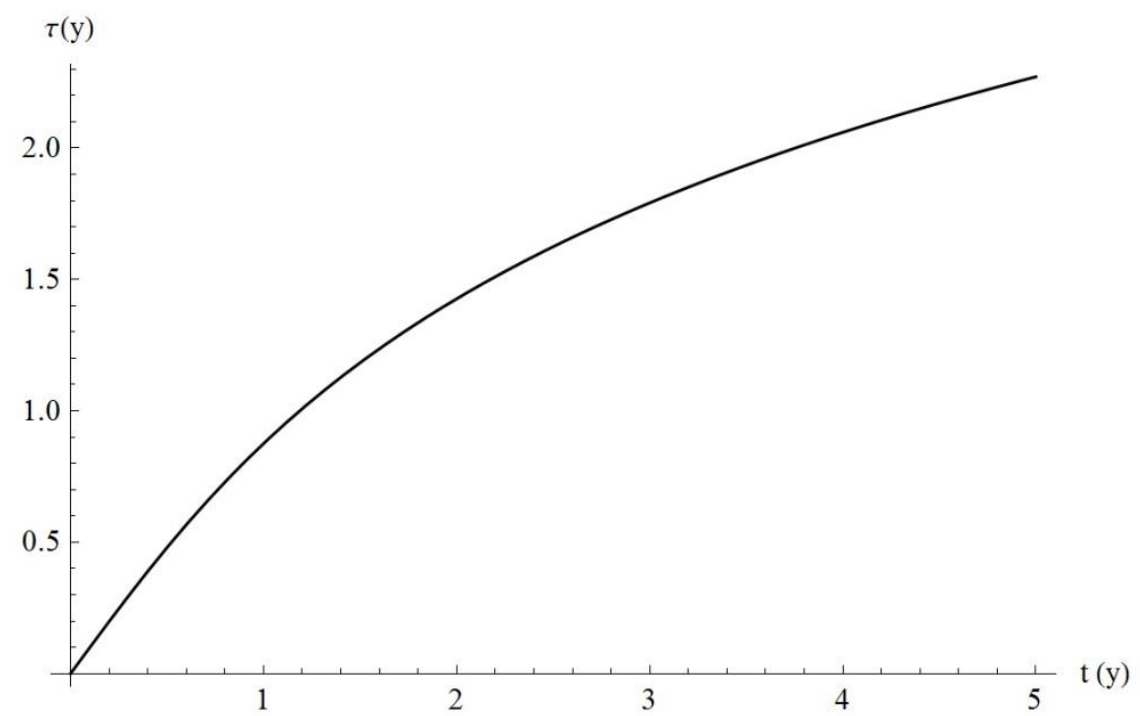

Figure 6. The proper time of the accelerating ship as a function of the time on Earth

What we would like to calculate, however, are the times for a round trip journey and how they compare to each other depending on how far the destination is. For this we will use that the journey is symmetric, i.e. the ship is gaining speed up to halfway $\left(x_{h}\right)$ to the destination $\left(d=2 x_{h}\right)$, then it slows down taking the same time as for the first half of the trip in both reference frames. The trip back home takes the same time as the trip to the destination in both reference frames, thus for the entire journey we can use that $\tau_{j}=4 \tau_{h}$ and $t_{j}=4 t_{h}$. Now we can express how the duration of the journey on the ship depends on how much time it takes on Earth (Figure 7), or we can also rearrange it the other way:

$$
\tau_{j}\left(t_{j}\right)=\frac{4 c}{g} \sinh ^{-1}\left(\frac{g t_{j}}{4 c}\right)
$$




$$
t_{j}\left(\tau_{j}\right)=\frac{4 c}{g} \sinh \left(\frac{g \tau_{j}}{4 c}\right)
$$

Using the result of (11) for the distance $x$, we can write how the distance of the reachable destination will depend on the time measured on Earth, or the other way, how the time of the journey will depend on how far the destination is:

$$
d\left(t_{j}\right)=\frac{2 c^{2}}{g}\left(\sqrt{1+\frac{g^{2}}{16 c^{2}} t_{j}^{2}}-1\right) \quad \text { or } \quad t_{j}(d)=\frac{2 d}{c} \sqrt{1+\frac{4 c^{2}}{d g}}
$$

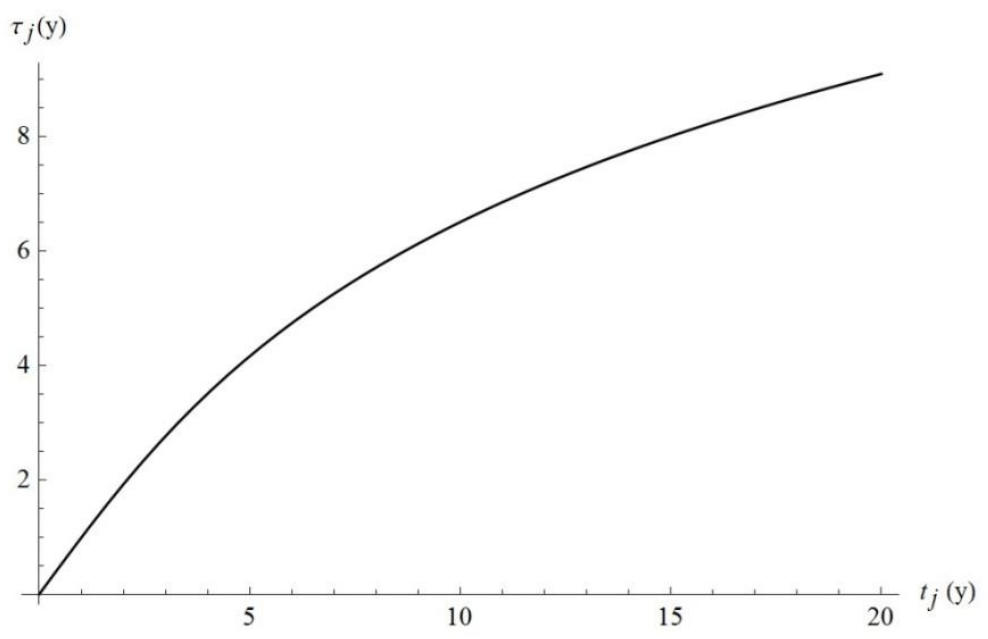

Figure 7. The duration of the journey as measured by the two twins

Finally, using the second expression in both (14) and (15), we can obtain a similar dependence between time and distance, but now with the proper time of the journey, as measured on the ship:

$$
d\left(\tau_{j}\right)=\frac{2 c^{2}}{g}\left(\sqrt{1+\sinh ^{2}\left(\frac{g \tau_{j}}{4 c}\right)}-1\right) \quad \text { or } \quad \tau_{j}(d)=\frac{4 c}{g} \sinh ^{-1}\left(\frac{g d}{2 c^{2}} \sqrt{1+\frac{4 c^{2}}{g d}}\right)
$$

\section{Conclusions}

Starting with the transformation formulas of the theory of special relativity, we managed to find analytical solutions for the times measured by the two twins depending on how far one of them travels on a continuously accelerating spaceship. If we want to see how much younger the traveling twin will be after the journey, we just have to take the difference of the two times, i.e. the second formulas of (15) and (16):

$$
t_{\Delta}(d)=t_{j}(d)-\tau_{j}(d)=\frac{2 d}{c} \sqrt{1+\frac{4 c^{2}}{d g}}-\frac{4 c}{g} \sinh ^{-1}\left(\frac{g d}{2 c^{2}} \sqrt{1+\frac{4 c^{2}}{g d}}\right)
$$


If we look at Figure 8 that shows the times measured on the Earth and on the spaceship, as well as the difference of these two times, as a function of the distance to the destination, we can see that for short journeys there are no time dilation effects, because the spaceship does not have enough time to accelerate to relativistic speeds. On the other hand, when the journey is to a destination that is several tens of light years away, the difference between the times becomes very big. For extremely long journeys (see Table 1) the time measured on Earth would practically be as if the spaceship was traveling at the speed of light there and back, while on the ship these times would be much shorter. Thus the time difference eventually becomes almost as large as the time measured on Earth.

Table 1. Times measured by the two twins for round trips to various destinations

\begin{tabular}{|c|c|c|c|c|}
\hline Destination & $\mathrm{d}[\mathrm{ly}]$ & $t_{j}[\mathrm{y}]$ & $\tau_{j}[\mathrm{y}]$ & $t_{\Delta}[\mathrm{y}]$ \\
\hline 1 light year & 1 & 4.42 & 3.79 & 0.63 \\
\hline Proxima & 4.3 & 11.86 & 7.12 & 4.74 \\
\hline Neighbourhood & 20 & 43.71 & 12.09 & 31.62 \\
\hline TOI 700 d & 100 & 203.8 & 18.05 & 185.8 \\
\hline Galactic centre & 26500 & 53004 & 39.61 & 52964 \\
\hline Andromeda & 2.5 million & 5 million & 57.24 & $\sim 5$ million \\
\hline
\end{tabular}

For example, as it can be seen from Table 1, even a round trip to the centre of the Milky Way, which is about 26500 light years away, would still take less than 40 years on the ship.

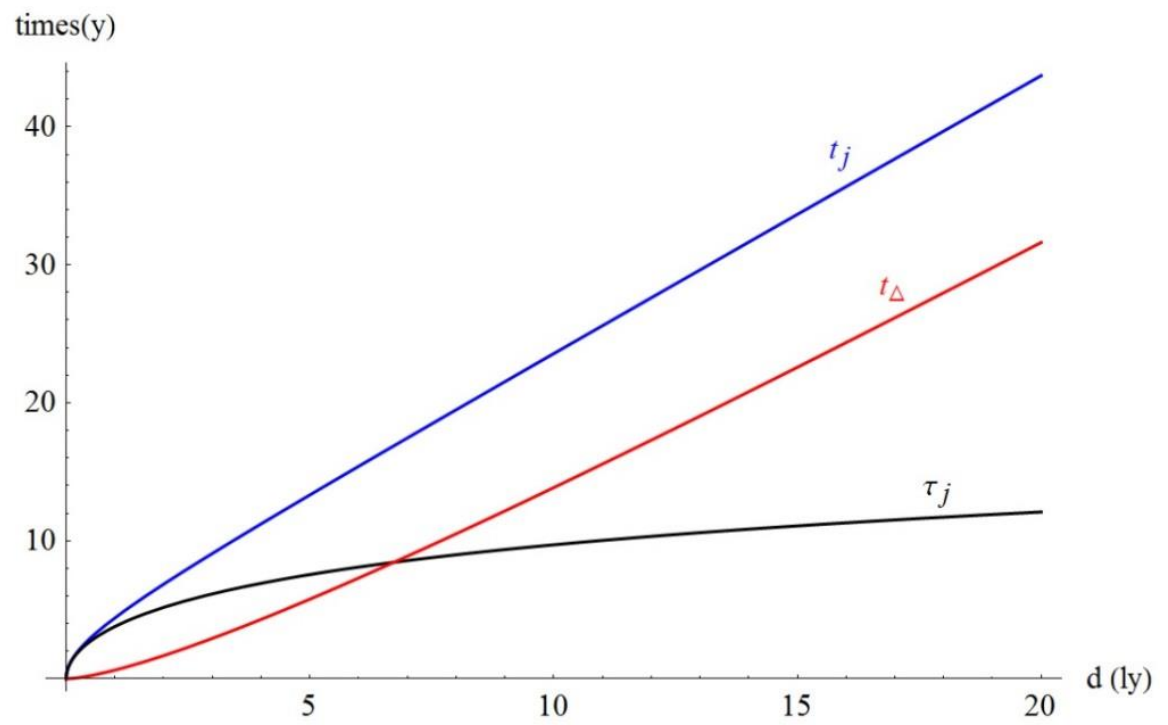

Figure 8. The durations measured by the two twins for round trips to various distances, and the difference between the two times (age difference). 
This means that there is a sure way for traveling not just to the distant stars, but also into the future, provided that this kind of interstellar travel becomes possible at some point.

\section{Acknowledgements}

The research reported here was carried out as part of the EFOP-3.6.1-16-2016-00011 "Younger and Renewing University - Innovative Knowledge City - Institutional development of the University of Miskolc aiming at intelligent specialization" project implemented in the framework of the Szechenyi 2020 program. The realization of this project is supported by the European Union, co-financed by the European Social Fund.

\section{References}

[1] Einstein, Albert, "Zur Elektrodynamik bewegter Körper", Annalen der Physik 322 (10): 891921. English translation: "On The Electrodynamics of Moving Bodies", (1905). https://doi.org/10.1002/andp.19053221004

[2] Serway, Raymond A.; Moses, Clement J.; Moyer, Curt A. Modern Physics (3rd ed.). Cengage Learning. p. 21. ISBN 978-1-111-79437-8. (2004).

[3] Baez, John. "Can Special Relativity Handle Acceleration?" (1996).

[4] http://math.ucr.edu/home/baez/physics/Relativity/SR/acceleration.htm

[5] J. C. Hafele and Richard E. Keating. Around-the-World Atomic Clocks: Observed Relativistic Time Gains. Science 1972, 177(4044):168-170. https://doi.org/10.1126/science.177.4044.168

[6] Rossi, Bruno; Hall, David B. Variation of the Rate of Decay of Mesotrons with Momentum. Physical Review 1941, 59(3):223-228. https://doi.org/10.1103/PhysRev.59.223

[7] Frisch, David H.; Smith, James H. Measurement of the Relativistic Time Dilation Using $\mu$ Mesons. American Journal of Physics 1963, 31(5):342-355. https://doi.org/10.1119/1.1969508

[8] Reinhardt, S.; et al. Test of relativistic time dilation with fast optical atomic clocks at different velocities, Nature Physics 2007, 3(12):861-864. https://doi.org/10.1038/nphys778 